\title{
Brain-Derived Neurotrophic Factor Differentially Regulates Retinal Ganglion Cell Dendritic and Axonal Arborization In Vivo
}

\author{
Barbara Lom and Susana Cohen-Cory \\ Mental Retardation Research Center, Departments of Psychiatry and Neurobiology, University of California, Los Angeles, \\ Los Angeles, California 90025
}

Expression of the neurotrophin brain-derived neurotrophic factor (BDNF) and its receptor trkB in the ganglion cell layer of the Xenopus retina during retinal ganglion cell (RGC) dendritic arborization indicates that BDNF is spatially and temporally available to influence RGC morphological differentiation (CohenCory and Fraser, 1994; Cohen-Cory et al., 1996). BDNF promotes RGC axon arborization in vivo by acting as a targetderived trophic factor (Cohen-Cory and Fraser, 1995). To determine whether BDNF also acts locally to regulate RGC dendritic development in vivo, we altered retinal neurotrophin levels at the onset of dendritic arborization and assessed the resulting arbor morphologies of RGCs retrogradely labeled with fluorescent dextrans. Injecting neurotrophins or BDNF functionblocking antibodies coupled to microspheres provided local alterations of retinal neurotrophin levels. BDNF significantly decreased RGC dendritic arbor complexity, whereas neutralizing endogenous BDNF levels with function-blocking antibodies significantly increased dendritic arbor complexity. RGCs ex- posed to other neurotrophins, as well as RGCs in retinae treated with BDNF but in areas not directly exposed to the neurotrophin, developed dendritic arbors that were indistinguishable from controls, indicating that exogenous BDNF acts specifically and locally. In the tectum, where RGC axons arborize, BDNF had opposite effects. BDNF significantly increased RGC axon arbor complexity and anti-BDNF reduced RGC arborization. Thus, BDNF reduces RGC dendritic arborization within the retina and increases axon arborization in the tectum. These results indicate that BDNF can differentially modulate axonal and dendritic arborization within a single neuronal population in opposing manners and raise the possibility that differential modulation by a neurotrophic factor finely tunes the morphological differentiation program of a neuron.

Key words: brain-derived neurotrophic factor; neurotrophins; retinal ganglion cell; visual system; dendrite; Xenopus laevis; axon; retina
For the nervous system to function properly, neurons must form intricate networks of precise synaptic connections that permit the transfer of information between individual neurons. Numerous developmental events, including neuronal survival, migration, arborization, and synaptic modification, must be coordinated spatially and temporally to assure that appropriate synapses are formed and maintained (for review, see Goodman and Shatz, 1993). The vertebrate visual system has long served as an accessible experimental model for investigating the cues and developmental events that contribute to neuronal differentiation. As the visual system develops, the morphologies of individual neurons change dramatically. The process of dendritic and axonal arborization is a dynamic combination of branch addition and branch elimination as well as branch lengthening and shortening (O'Rourke et al., 1994; Cohen-Cory and Fraser, 1995; Witte et al., 1996). It is thought that the dynamic changes in arbor morphology reflect the discrete formation, stabilization, and elimina-

\footnotetext{
Received June 24, 1999; revised Sept. 3, 1999; accepted Sept. 3, 1999.

This work was supported by an National Eye Institute (NEI) postdoctoral fellowship to B.L. and awards from the NEI, Alfred P. Sloan, Stein/Oppenheimer, UCLA Frontiers of Science, and Beckman Foundations to S.C-C. We thank Ami Poon for developing preliminary dextran-labeling protocols; Tilly Oren, Thuy $\mathrm{Vu}$, and Ly Nguyen for technical assistance; and Drs. Ron Frostig and Jeff Cogen for providing insightful comments on this manuscript. Amgen (Thousand Oaks, CA) and Genentech (South San Francisco, CA) generously provided the neurotrophins used in this study.

Correspondence should be addressed to Dr. Susana Cohen-Cory, University of California, Los Angeles, MRRC, 760 Westwood Plaza, NPI 78-148, Los Angeles, CA 90095. E-mail: scohenco@ucla.edu.

Copyright (C) 1999 Society for Neuroscience 0270-6474/99/199928-11\$05.00/0
}

tion of the synaptic connections of a neuron. Thus, understanding how developmental cues sculpt neuronal architecture can provide valuable insight into the mechanisms necessary to establishing a functional nervous system.

One family of molecular cues, the neurotrophins, has been shown to play significant roles in diverse aspects of nervous system development from the control of cell survival to the modulation of synaptic efficacy (Barde, 1994; Berninger and Poo, 1996; Snider and Lichtman, 1996; McAllister et al., 1999). The neurotrophin family includes the ligands brain-derived neurotrophic factor (BDNF), nerve growth factor (NGF), neurotrophin-3 (NT-3), and neurotrophin-4 (NT-4) and their specific tyrosine kinase receptors (Barbacid, 1995; Bothwell, 1995). In the visual system the neurotrophins are potent modulators of neuronal architecture and survival. Neurotrophins can rescue retinal neurons in a target-dependent manner (Rodriguez-Tebar et al., 1989) (for review, see von Bartheld, 1998) and can enhance neurite outgrowth in vitro (for review, see von Bartheld, 1998; McAllister et al., 1999). In vivo, neurotrophins have been shown to play a role in shaping neuronal morphology by acting as target-derived trophic factors (Cabelli et al., 1995; Cohen-Cory and Fraser, 1995; Riddle et al., 1995). One direct example of this is the role of BDNF during retinal ganglion cell (RGC) axon arborization (Cohen-Cory and Fraser, 1995). Increasing endogenous tectal BDNF can promote RGC axon arborization in live Xenopus tadpoles, whereas neutralizing endogenous BDNF decreases axon arborization (Cohen-Cory and Fraser, 1995). The peak in BDNF expression in the Xenopus visual system coincides with the 
period of active RGC axon arborization at its target (Cohen-Cory and Fraser, 1994), providing further support for BDNF as a target-derived modulator of axon morphology.

In addition to its target-derived roles, BDNF may act as a local modulator of neuronal morphology. In the visual system of most vertebrate species BDNF is expressed at the target optic tectum as well as locally within the retina (Cohen-Cory and Fraser, 1994; Perez and Caminos, 1995; Cohen-Cory et al., 1996; Hallbook et al., 1996) (for review in greater detail, see von Bartheld, 1998), and RGCs express trkB, the specific receptor for BDNF (Jelsma et al., 1993; Cohen-Cory and Fraser, 1994; Escandon et al., 1994; Garner et al., 1996). In the Xenopus retina, BDNF and trkB are expressed by RGCs during the period of active RGC dendritic elaboration (Cohen-Cory et al., 1996), indicating that BDNF is spatially and temporally available to influence an RGC's own morphological differentiation program. Now we have investigated the role of retinal-derived BDNF during RGC morphological development in vivo by experimentally altering retinal neurotrophin levels during dendritic arborization in live Xenopus laevis tadpoles and then assessing the resulting RGC arbor morphologies. Applications of exogenous BDNF decreased RGC dendritic arbor complexity, whereas neutralizing endogenous BDNF with function-blocking antibodies increased dendritic arbor complexity. These results contrast with the ability of BDNF to promote RGC axon arborization at the tectum, indicating that within a single neuronal population BDNF can modulate axonal versus dendritic arborization in distinct and opposing manners. Thus, by acting locally or at the target, a single neurotrophin can differentially modulate the morphological differentiation of a neuron.

\section{MATERIALS AND METHODS}

Xenopus laevis tadpoles. Xenopus laevis embryos were obtained by in vitro fertilization of eggs obtained from females primed with human chorionic gonadotropin (Sigma, St. Louis, MO). Embryos were reared in a 20\% modified Steinberg's solution [containing (in mM) $60 \mathrm{NaCl}, 0.67 \mathrm{KCl}$, $0.34 \mathrm{Ca}\left(\mathrm{NO}_{3}\right)_{2}, 0.83 \mathrm{MgSO}_{4}$, and 10 HEPES, pH 7.4 (Keller, 1991)] supplemented with $40 \mathrm{mg} / 1$ gentamycin (Sigma). Embryos were staged developmentally according to Nieuwkoop and Faber (1956). To reduce pigmentation, we added $0.001 \%$ phenylthiocarbamide (Sigma) to the rearing solution $\sim 1 \mathrm{~d}$ after fertilization. During experimental injections and observations the tadpoles were anesthetized by immersion in $0.05 \%$ tricane methanesulfonate (Finquel, Argent Laboratories, Redmond, WA) in rearing solution.

Neurotrophin-coated fluorescent microspheres. Green fluorescent microspheres of 50-200 nm diameter (Lumafluor, New York, NY) were prepared and coated with neurotrophins in a manner similar to that described by Riddle et al. (1997). Microspheres were incubated overnight at $4^{\circ} \mathrm{C}$ in a $1: 5 \mathrm{mix}$ of microspheres and $100 \mathrm{ng} / \mu \mathrm{l}$ neurotrophin solution, a concentration that has been used effectively both in vitro and in vivo (Riddle et al., 1997). After coating, the microspheres were centrifuged at $14,000 \times g$ for $30 \mathrm{~min}$ and resuspended in sterile water for a final $10 \%$ microsphere concentration. BDNF was a gift from Amgen (Thousand Oaks, CA), and NT-3, NT-4, and NGF were gifts from Genentech (South San Francisco, CA). Neurotrophin-coated microspheres exert neurotrophic activity comparable to that of free neurotrophins that can be retained for at least $4 \mathrm{~d}$ (Riddle et al., 1997). Anti-BDNF (R \& D Systems, Minneapolis, MN) was mixed with deionized microspheres to a concentration of $250-375 \mu \mathrm{g} / \mathrm{ml}$ immediately before injection. We observed that $100 \mu \mathrm{g} / \mathrm{ml}$ of this antibody completely inhibited survival in dorsal root ganglion neurons normally elicited by $2.5 \mathrm{ng} / \mathrm{ml} \mathrm{rhBDNF}$ in vitro and showed $<2 \%$ cross-reactivity with NGF, NT-3, and NT-4, as demonstrated by ELISA and Western assays (R \& D Systems). Control microspheres were incubated with no protein, as described above, with $100 \mathrm{ng} / \mu \mathrm{l}$ cytochrome $c$ (Sigma), or with $100 \mu \mathrm{g} / \mathrm{ml}$ of nonimmune IgG. Neurotrophin-, antibody-, or control-coated microspheres were pressureinjected into the right eyes of anesthetized stage 38 tadpoles. Then the tadpoles were reared in the dark until they reached stage $42-43(\sim 1-1.5$ d later).

$R G C$ labeling. Rhodamine-conjugated dextran or biotin-conjugated dextran (3 kDa molecular weight; Molecular Probes, Eugene, OR) was used to retrogradely label RGCs. Dextrans were diluted in sterile water to $100 \mathrm{mg} / \mathrm{ml}$, purified by centrifugation in a $3 \mathrm{kDa}$ cutoff microconcentrator (Amicon, Beverly, MA), and pressure-injected into the left tecta of anesthetized stage $42-43$ tadpoles. Then the tadpoles were allowed to develop to stage $45(\sim 1 \mathrm{~d}$ later $)$ in the dark. The tadpoles were fixed in $4 \%$ paraformaldehyde with $4 \%$ sucrose overnight at $4{ }^{\circ} \mathrm{C}$ and rinsed in PBS. The retinae of tadpoles injected with rhodamine-dextran were prepared as whole mounts. The pigment epithelium and lens were removed carefully from isolated right eyes; then the retinae were dehydrated by immersion in a graded series of ethanol. Small cuts in the peripheral retina were made so that the tissue could be mounted flat on a slide with the RGC layer up and coverslipped with Krystalon mounting media (EM Industries, Gibbstown, NJ). Cryostat sections $(20 \mu \mathrm{m})$ of tadpoles injected with biotinylated dextran were stained by the $\mathrm{ABC}$ method (Vector Laboratories, Burlingame, CA). Tectal injections of rhodamine- or biotin-dextran randomly filled a subpopulation of RGCs, with soma distributed throughout the retinal ganglion cell layer at sufficiently sparse densities that individual dendritic arbors were discriminated easily. No other retinal cell type was labeled by this procedure (see Fig. 1A).

Arbor analysis. Retinal whole mounts were coded so that RGCs were traced and analyzed without knowledge of the treatment type. Only dendritic arbors of rhodamine-labeled RGCs that were surrounded by fluorescent microspheres were evaluated. The only exception to this were rhodamine-labeled RGC arbors that did not coincide with observable BDNF-treated microspheres. These RGCs were analyzed as a distinct category, termed noncoincident (NC). Analysis of NC RGCs served as an internal control to determine whether exogenous BDNF exerted effects beyond its delineated injection site. NC RGCs were positioned at least $100 \mu \mathrm{m}$ away from the nearest BDNF-treated microspheres. Previous work has indicated that these microspheres do not diffuse significantly from their injection site in vivo (Riddle et al., 1997) and thereby provide local sources of neurotrophic factors.

Dendritic morphology was observed by epifluorescent microscopy (Nikon, Tokyo, Japan). A $0.5 \mu \mathrm{m}$ interval $z$-series was captured throughout the extent of the dendritic arbor of the RGC with a CCD camera (Photometrics, Tucson, AZ) controlled by MetaMorph software (Universal Imaging, West Chester, PA). The dendritic arbor of each RGC was traced manually on transparent acetate film to represent the dendritic arbor in two dimensions. Tracings were scanned digitally and then analyzed with MetaMorph imaging software.

Primary dendrites were defined as direct extensions from the soma of at least $10 \mu \mathrm{m}$ in length. Only RGCs with at least one primary dendrite $>10 \mu \mathrm{m}$ in total length were analyzed. Branch tips were identified as the terminal end(s) of primary dendrites. To calculate soma area and total dendritic arbor length, we thresholded, binarized, and skeletonized images with MetaMorph software so that the soma perimeter and dendrites were represented as a single pixel width. To determine dendritic arbor complexity, we assigned each branch tip an order value that equaled the number of branch points between the tip and the base of its primary dendrite (also see Fig. 6A). A dendritic complexity index (DCI) was calculated for each RGC such that DCI $=[$ (sum of branch tip orders + number of branch tips)(total arbor length)/(number of primary dendrites)] (also see Fig. 6). To correct for small variabilities in developmental staging, we normalized values to the average control value of each parameter for each of five independent experiments. All measurements were compared by ANOVA, including Tukey's post hoc test (Systat, SPSS, Chicago, IL). Significance was assigned when $p<0.05(*), p<$ $0.01\left(^{* *}\right)$, or $p<0.001\left(^{* * *}\right)$. Morphological analysis revealed that RGCs developing in the presence of cytochrome $c$-coated beads were indistinguishable from RGCs developing in the presence of uncoated beads; thus controls include both groups. Microspheres coated with bovine serum albumin (BSA) or nonimmune serum served as controls to establish the specificity of the anti-BDNF effects on RGC dendritic morphology. RGCs exposed to BSA or to nonimmune IgG-coated beads resembled RGC exposed controls (data not shown), indicating that the antiBDNF effects were specific and not attributable to other proteins or immunoglobulins present in the serum.

RGC axon arbors were visualized as described in Cohen-Cory and Fraser (1995). Briefly, the retinae of anesthetized stage 41 tadpoles were injected with minute amounts of the fluorescent vital dye DiI (Molecular Probes) to label RGCs. Tadpoles with one to two distinguishable axonal arbors branching in the optic tectum were imaged with a Bio-Rad (Richmond, CA) laser-scanning confocal microscope before and $24 \mathrm{hr}$ 

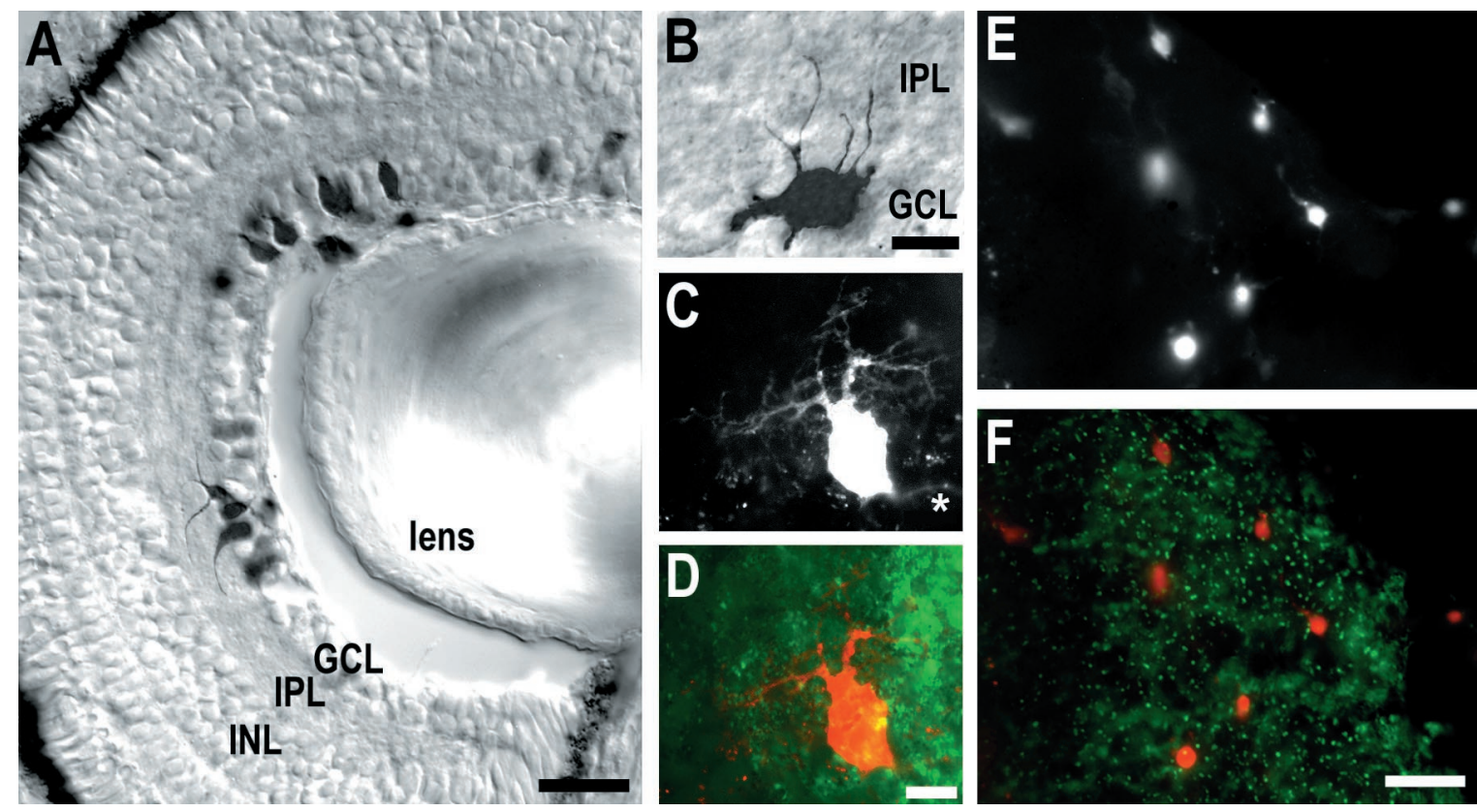

Figure 1. Visualization of RGC dendritic arbors with labeled dextrans. To visualize RGC dendritic arbor morphologies, we injected biotin- or rhodamine-conjugated dextrans into the tecta of Xenopus tadpoles during developmental stages when RGC axons are arborizing actively in the optic tectum. Retrograde transport of dextran specifically labels RGC soma and dendrites within the retina. $A, B$, Differential interference contrast images of transverse $20 \mu \mathrm{m}$ retina cryostat sections of a stage 45 Xenopus tadpole that had received tectal injection of biotin-conjugated dextran. $A$, Labeled neurons are distributed throughout the retinal ganglion cell layer $(G C L)$ and extend dendrites up into the inner plexiform layer (IPL). $B, \mathrm{~A}$ higher power view of a biotinylated dextran-labeled RGC extending its dendrites into the IPL. $C$, Fluorescent image of one optical section of a rhodamine-dextran-labeled RGC in a whole-mounted stage 45 Xenopus retina, and $(D)$ a corresponding overlay image showing both RGC morphology (red) and coincident NGF-coated microspheres (green). In $C$, the asterisk denotes the axon process. $E$, Low-power view of part of a retina whole mount showing rhodamine-dextran-labeled RGCs, and $(F)$ a corresponding overlay image showing both the retrogradely labeled RGCs (red) and the distribution of BDNF-coated beads in that area of the retina whole mount. $I N L$, Inner nuclear layer. Scale bars: $A, F, 50 \mu \mathrm{m} ; B, D, 10 \mu \mathrm{m}$.

after tectal application of BDNF or anti-BDNF. Vehicle solution $(10 \%$ Niu-Twitty), $200 \mathrm{ng} / \mathrm{ml}$ BDNF (Amgen), $200 \mu \mathrm{g} / \mathrm{ml}$ of specific functionblocking antibody to BDNF [a gift from J. Carnahan, Amgen; antibody specificity described in Nawa et al. (1995) and Ghosh et al. (1994)], or 200 $\mu \mathrm{g} / \mathrm{ml}$ of nonimmune $\mathrm{IgG}$ was pressure-injected into the subpial space overlying the caudal tectum immediately after the first observation. Delivery of neurotrophins or antibodies in solution provided a more reliable delivery mechanism of these reagents into the optic tectum than injection of the neurotrophin or antibody coupled to microspheres. This is attributable to the rapid dispersion of both internalized and free microspheres that results from active tectal cell proliferation and arbor refinement normally occurring at this developmental stage (J. Cogen and S. Cohen-Cory, unpublished observations). Axon arbor morphology and statistical analysis were performed as described in Cohen-Cory and Fraser (1995).

\section{RESULTS}

\section{BDNF inhibits RGC primary dendrites}

As reported previously, exogenous BDNF enhances Xenopus RGC axonal branching, and neutralization of tectal BDNF reduces RGC axon arborization in vivo (Cohen-Cory and Fraser, 1995) (also see Fig. 7). Thus, it is plausible that retinal BDNF also influences RGC dendritic branching locally. To determine whether retinal BDNF modulates RGC morphological differentiation in vivo, we experimentally altered neurotrophin levels in developing Xenopus retinae during the period when RGCs begin to differentiate (stage 38) and extend short unbranched primary dendrites within the inner plexiform layer of the retina (Sakaguchi et al., 1984; Holt, 1989). Microspheres treated with human recombinant neurotrophins or with function-blocking BDNF antibodies were injected directly into the right eyes of live anesthetized tadpoles to alter retinal neurotrophin levels. Binding neu- rotrophins to fluorescent microspheres prevented the neurotrophins from diff using from the delivery site and enabled us to analyze only those RGCs within the treatment site. RGCs were labeled retrogradely with rhodamine-dextran at stage 4243 , and tadpoles were reared until stage 45 , the developmental stage when endogenous retinal BDNF levels are maximal. This labeling protocol allowed a random population of newly arborizing RGC axon terminals to incorporate the dextran and retrogradely transport it back to the RGC soma and dendrites. This allowed us to visualize RGC dendritic arbors and trace their morphology (Figs. 1, 2).

Qualitatively, RGCs that developed in regions of exogenous BDNF appeared less complex than did RGCs exposed to other neurotrophins or controls, whereas RGCs that were exposed to BDNF-neutralizing antibodies exhibited more complex dendritic arbors (Fig. 2). To compare the influence of altered retinal neurotrophin levels on RGC dendritic arborization, we quantified several morphological parameters. The number of primary dendrites per RGC was evaluated by counting the dendrites that extended directly from the soma of each rhodamine-dextranlabeled RGC (Fig. 3A). RGCs that developed in retinal areas exposed to exogenous BDNF exhibited significantly fewer primary dendrites $(\mathrm{BDNF}=61 \pm 3.6 \%)$ than RGCs exposed to control microspheres (control $=100 \pm 4.9 \%$ ). To determine whether BDNF alters RGC dendritic number only on neurons directly exposed to the neurotrophin, we evaluated the primary dendrites of RGCs located in retinal areas devoid of BDNFcoated fluorescent microspheres. Such RGCs were termed BDNF noncoincident (NC) and resembled control-treated 
CONTROL

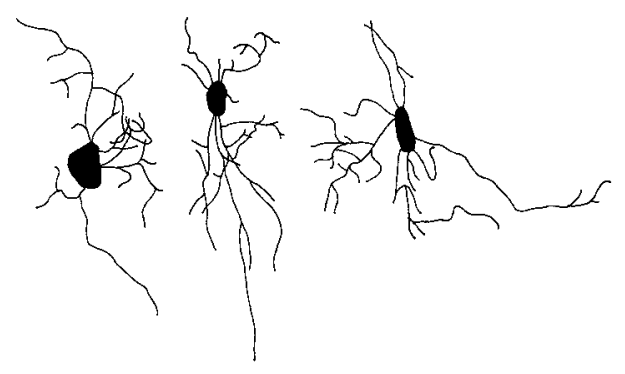

BDNF
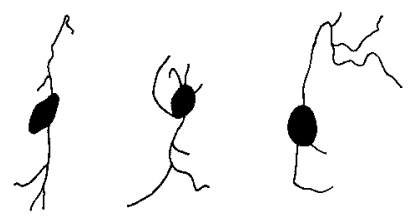

anti-BDNF
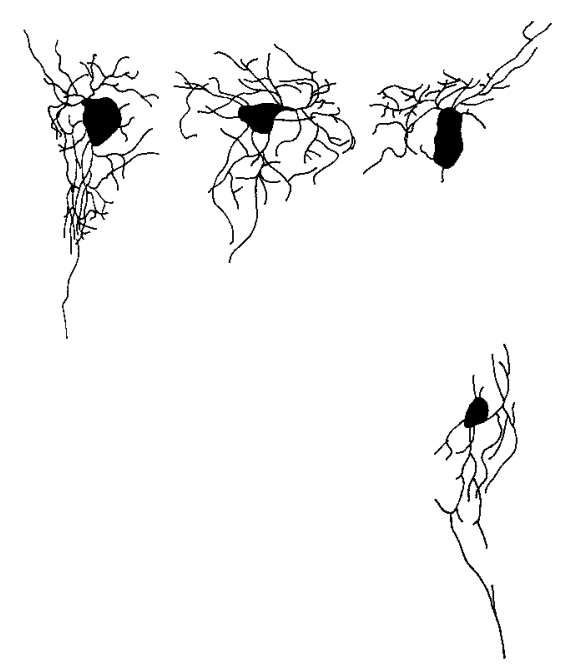

\section{NC}

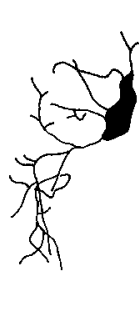

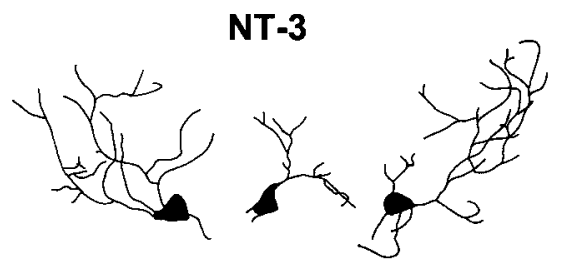

NT-4

NGF

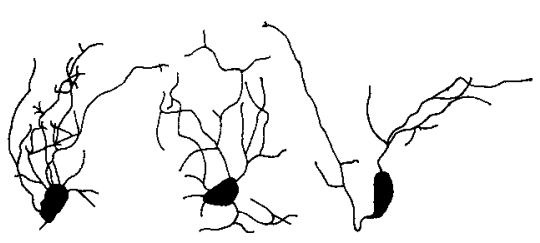

NT-3
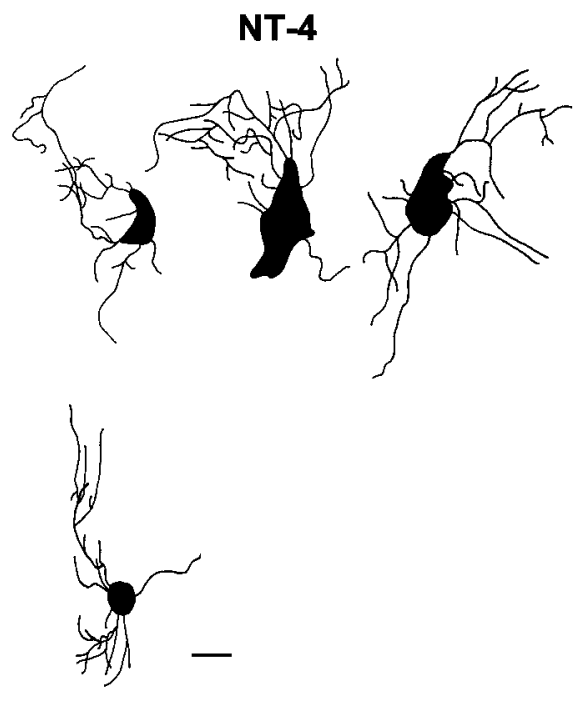

Figure 2. Neurotrophins modulate RGC dendritic complexity in vivo. Retinal neurotrophin levels were altered in vivo by injecting fluorescent microspheres treated with exogenous neurotrophin (BDNF, NGF, NT-3, or NT-4) to increase retinal levels or with function-blocking BDNF antibodies to neutralize endogenous BDNF (anti$B D N F$ ). Control treatments were injections of uncoated, cytochrome $c$-coated, or nonimmune IgG-coated microspheres. RGCs that developed in BDNF-treated retina, but noncoincident $(N C)$ with the exogenous neurotrophin, were analyzed also. The morphologies of rhodaminedextran-labeled RGCs were reconstructed from serial optical sections into one plane to quantify morphological parameters that describe dendritic arborization, such as primary dendrite number and branch tip number (see Figs. 3-6). RGCs are depicted in whole mounts viewed from the inner, or vitreal, surface of the retina, and axons are not shown. For control RGCs the averages equaled four to five primary dendrites and $16-20$ branch tips, with the highest branch tip order of 4-5 and a 136-175 $\mu \mathrm{m}$ dendrite length. An overall dendritic complexity index (DCI) was calculated by combining several of these morphological measures (see Fig. 6 and Materials and Methods for explanation of the calculation). The control RGCs depicted in this figure had DCI values that ranged from 2777 to 2899 ; NC DCI values ranged from 2417 to 2807 ; BDNF DCI values ranged between 186 and 276; anti-BDNF DCI values ranged from 5851 to 7429 ; NGF DCI values ranged from 2705 to 3204 ; NT-3 DCI values ranged from 2220 to 2588 ; and NT-4 DCI values ranged from 2213 to 4282 . Scale bar, $10 \mu \mathrm{m}$.
RGCs. The average number of primary dendrites per NC RGC was indistinguishable from that for RGCs in retinae injected with control microspheres $(\mathrm{NC}=105 \pm 6.0 \%$ vs control $=100 \pm$ $4.9 \%$ ). This result indicates that neurotrophic regulation of RGC primary dendrite number is restricted spatially. Consequently, exogenous BDNF applications significantly inhibited primary dendrites only in the treatment site.

If BDNF specifically modulates RGC dendritic elaboration in vivo, then neutralizing endogenous retinal BDNF should alter the number of primary dendrites also. Indeed, RGCs that developed in retinal regions treated with BDNF function-blocking antibodies had significantly more primary dendrites than did control neurons (anti-BDNF $=135 \pm 5.7 \%$ vs control $=100 \pm 4.9 \%$; Fig. $3 A$ ). Thus, retinal applications of BDNF and anti-BDNF had opposing local effects on the number of primary dendrites per RGC, implicating endogenous retinal BDNF as a mediator of RGC primary dendritic development.

\section{BDNF influences RGC dendritic branching}

Our initial analysis indicated that perturbing endogenous retinal BDNF levels significantly altered RGC primary dendrite number.
To determine whether BDNF affects dendritic arbor differentiation exclusively by reducing the number of primary dendrites or whether BDNF also influences dendritic branching, we determined the total numbers of dendritic branch tips per RGC in retinae injected with control-, BDNF-, or anti-BDNF-treated microspheres. RGCs developing in the presence of exogenous BDNF had significantly fewer dendritic branch tips than RGCs developing in the presence of control microspheres $(\mathrm{BDNF}=$ $38 \pm 4.8 \%$ vs control $=100 \pm 7.2 \%$; Fig. $3 B$ ). NC RGCs branched as much as RGCs developing near control microspheres $(\mathrm{NC}=100 \pm 8.8 \%$ vs control $=100 \pm 7.2 \%)$. These results indicated that, for BDNF to affect branch tip number, RGCs had to be exposed directly to BDNF-coated microspheres.

Similar to its effects on primary dendrite extension, neutralizing endogenous retinal BDNF with anti-BDNF significantly altered branch tip number per RGC (Fig. 3B). RGC dendritic arbors had significantly more branch tips after exposure to antiBDNF than did control RGCs (anti-BDNF $=177 \pm 12 \%$ vs control $=100 \pm 7.2 \%$ ). These results indicate that increasing endogenous BDNF during active dendritic arborization de- 
A

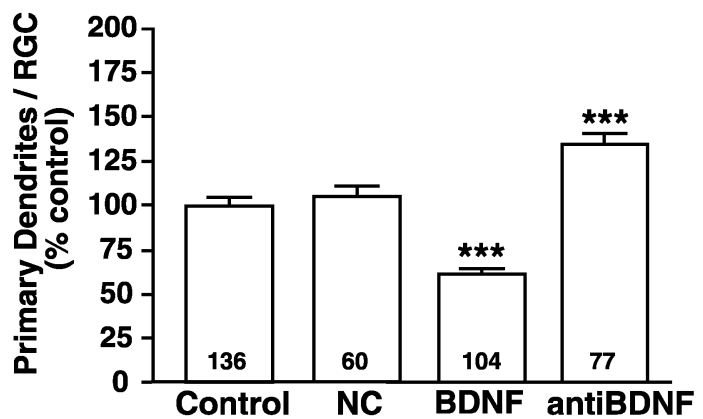

C

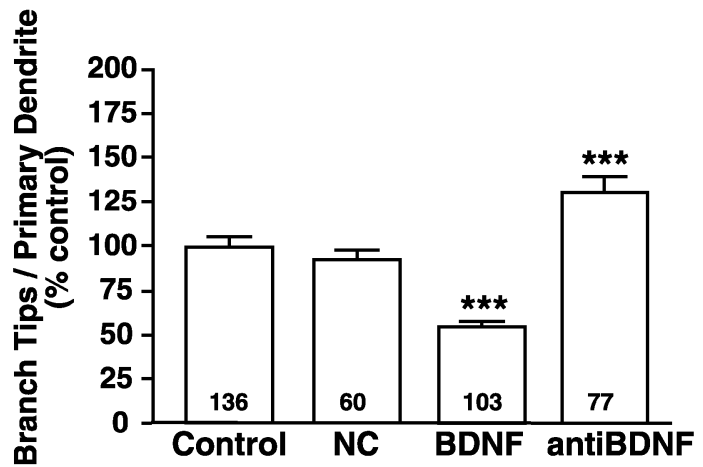

B

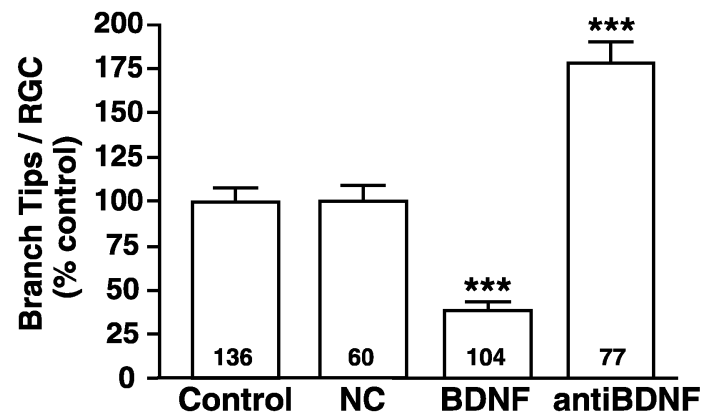

D

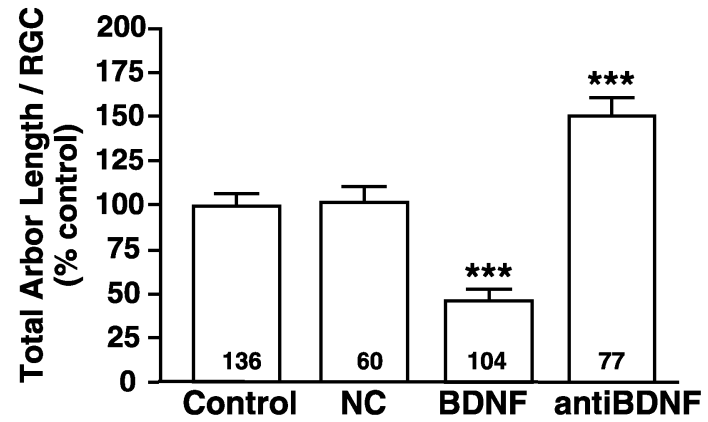

Figure 3. Retinal BDNF levels influence RGC dendritic arborization in vivo. Retinal neurotrophin levels were altered in vivo by exposure to exogenous $\mathrm{BDNF}$ to increase retinal BDNF or by exposure to function-blocking BDNF antibodies to neutralize endogenous BDNF during RGC dendritic elaboration. RGCs noncoincident $(N C)$ with BDNF-coated microspheres as well as RGCs coincident with control microspheres were analyzed also. Primary dendrite extension was evaluated by determining the number of primary dendrites per RGC $(A)$. Secondary dendritic branching was evaluated by determining the total number of branch tips per RGC $(B)$ as well as the number of branch tips per primary dendrite $(C)$. The extent of dendritic elaboration also was compared by measuring the total dendritic arbor length $(D)$. Primary dendritic number $(A)$, number of dendritic branch tips $(B$, $C$ ), and total arbor length $(D)$ were decreased significantly by exogenous BDNF and increased significantly by neutralizing BDNF. RGCs not coincident $(N C)$ with BDNF-coated microspheres did not differ from controls in any of these morphological parameters. Plotted values represent the averages from five independent experiments; error bars indicate \pm SEM. Numbers within the bars indicate the total number $(n)$ of RGCs analyzed for each treatment. $* * * p<0.001$ as compared with control.

creased total dendritic branch tip number, and neutralizing endogenous retinal BDNF significantly increased arbor branching. These results support the idea that endogenous retinal BDNF plays a significant role in modulating both RGC primary dendrite extension and branch number.

To determine whether the negative regulation of BDNF branch tip number was a sole consequence of the reduction of BDNF in primary dendrite number or whether the reduced number of branch tips per RGC was also attributable to decreased secondary branching, we calculated the average number of branch tips per primary dendrite (Fig. 3C). RGCs coincident with BDNF-coated microspheres had significantly fewer branch tips per primary dendrite than did RGCs coincident with control microspheres $(\mathrm{BDNF}=52 \pm 3.0 \%$ vs control $=100 \pm 5.9 \%)$. The average number of branch tips per primary dendrite of NC RGCs was not significantly different from control-treated RGCs $(\mathrm{NC}=95 \pm 6.5 \%$ vs control $=100 \pm 5.9 \%)$. In addition, RGCs that developed near microspheres treated with anti-BDNF exhibited significantly more branch tips per primary dendrite than did controls, indicating that endogenous BDNF influences RGC dendritic branching (anti-BDNF $=129 \pm 8.2$ vs control $=100 \pm$ $5.9 \%$ ). Thus, the opposing effects that exogenous BDNF and anti-BDNF exert on both total branch tip number per RGC and branch tips per primary dendrite suggest that endogenous BDNF modulates both the extension of primary dendrites and their subsequent branching.

\section{BDNF reduces RGC total dendritic arbor length}

The extent of a dendritic arbor of a neuron is thought to influence its potential for receiving presynaptic input. We observed that increasing retinal BDNF levels decreased both the number of RGC primary dendrites and their subsequent branching. Thus, it is possible that a neuron could compensate for decreased dendrite number and branching by extending longer dendrites in an attempt to achieve a targeted total length of its dendritic arbor. To determine whether alterations in retinal BDNF levels influenced dendritic arbor length, we compared the total arbor length of RGCs exposed to BDNF- or anti-BDNF-coated microspheres with RGCs exposed to control microspheres (Fig. 3D). BDNF significantly reduced total dendritic arbor length as compared with control RGCs (BDNF $=47 \pm 6.4 \%$ vs control $=100 \pm$ 7.4\%). RGCs noncoincident with BDNF-coated microspheres extended arbors of equivalent length to controls $(\mathrm{NC}=102 \pm$ $8.6 \%$ vs control $=100 \pm 7.4 \%$ ). RGCs in retinae exposed to BDNF antibodies had significantly larger dendritic arbors (anti$\mathrm{BDNF}=151 \pm 10 \%$ vs control $=100 \pm 7.4 \%)$. Thus, total 
dendritic arbor length is decreased by exogenous BDNF and is increased by neutralizing endogenous BDNF to values similar to those for primary dendrite number. Moreover, the observation that the total dendritic arbor length of RGCs exposed to control microspheres was indistinguishable from that of noncoincident RGCs again supports our observations that the effects of BDNF within the retina are local. The decrease in total arbor length of BDNF-treated RGCs results from reductions in primary dendrite formation and secondary branching without significant compensatory increase of dendritic length.

\section{RGC dendritic arborization is unaffected by neurotrophins other than BDNF}

To determine whether BDNF specifically regulates RGC dendritic arborization, we similarly increased retinal levels of other neurotrophins by injecting microspheres coated with NGF, NT-3, or NT-4 into developing Xenopus retinae. Dendritic morphologies of RGCs exposed to elevated concentrations of NGF, NT-3, or NT-4 were compared with RGCs exposed to BDNF- or control-treated microspheres. Unlike BDNF, the neurotrophins NGF, NT-3, and NT-4 did not alter significantly the number of primary dendrites per RGC (Fig. $4 A$ ). Similarly, the number of branch tips per primary dendrite did not differ from controls in RGCs exposed to elevated levels of NGF, NT-3, or NT-4 (Fig. $4 B$ ), indicating that neurotrophins other than BDNF do not affect either primary dendrite number or dendritic branching. These results suggest that the influence of BDNF on dendritic complexity is not a general neurotrophic response but, rather, a specific response to $\mathrm{BDNF}$.

\section{Neurotrophins influence RGC soma size independently of dendritic arborization}

To determine whether the reduction by BDNF of RGC dendritic morphology was attributable to a general effect on neuronal differentiation, we measured and compared RGC soma area for all neurotrophin treatments (Fig. $5 A$ ). Neither exposure to exogenous BDNF $(\mathrm{BDNF}=90 \pm 5.7 \%)$ nor to anti-BDNF (anti$\mathrm{BDNF}=112 \pm 6.5 \%$ ) significantly altered $\mathrm{RGC}$ soma area when compared with controls (control $=100 \pm 4.3 \%$ ). As observed for dendritic parameters, RGCs that developed in retinae injected with BDNF-coated microspheres, but in areas noncoincident with the microspheres, resembled RGCs exposed to control microspheres $(\mathrm{NC}=98 \pm 7.3 \%)$. Thus, neither the reduction of BDNF nor the increase of anti-BDNF in dendritic elaboration parameters results from general alterations in cell size. Although NGF, NT-3, and NT-4 did not alter RGC dendritic morphological parameters significantly, NT-3 significantly reduced soma size (72 $\pm 5.6 \%)$ and NT-4 significantly increased RGC soma size (142 \pm $12 \%)$, as compared with control-treated retinae (100 $\pm 4.3 \%$; Fig. $5 B)$. Neither BDNF nor NGF significantly affected soma area. Thus, neurotrophic influences on RGC soma size suggest that individual neurotrophins exert distinct biological effects on various aspects of RGC development. Neurotrophic regulation of soma area, therefore, is likely to be independent from the regulation of dendritic morphology.

\section{Neurotrophins differentially regulate RGC dendritic complexity}

To compare overall dendritic morphology between RGCs that developed in altered neurotrophic environments, we calculated a dendritic complexity index (DCI) for each RGC to describe RGC complexity numerically (Fig. 6A,B). RGCs exposed to BDNF, NGF, NT-3, NT-4, anti-BDNF, or control microspheres dis-
A

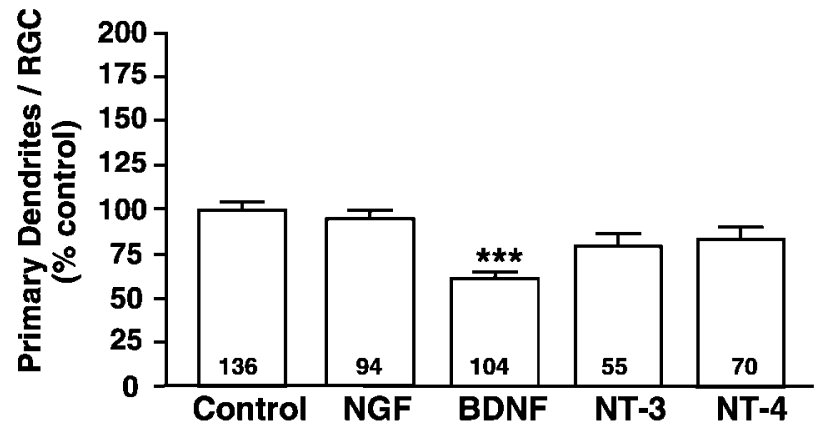

B

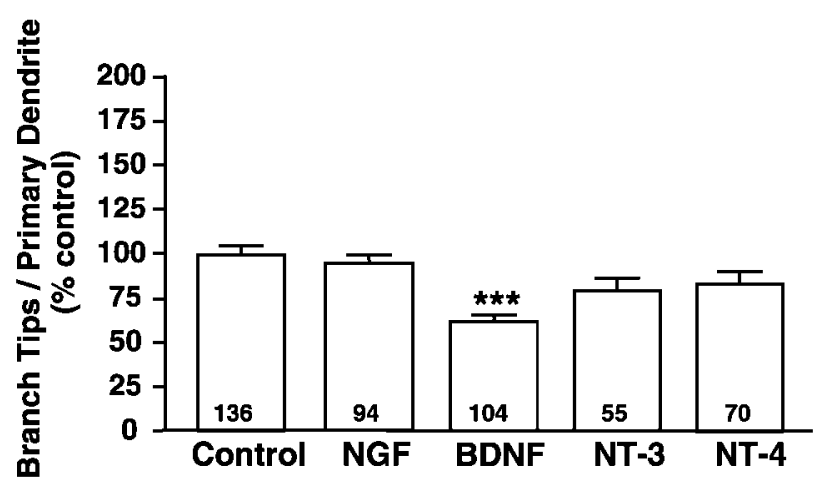

Figure 4. BDNF specifically influences RGC dendritic arborization in vivo. Retinal neurotrophin levels were increased in vivo by injecting fluorescent microspheres coated with control protein, BDNF, NGF, NT-3, or NT-4; the resulting dendritic arbors of rhodamine-dextranlabeled RGCs were visualized. Primary dendritogenesis was evaluated by counting the number of primary dendrites per RGC $(A)$, whereas dendritic branching was evaluated by measuring the number of branch tips per primary dendrite $(B)$. Only exogenous BDNF treatment significantly influenced primary dendrite extension $(A)$ or branching $(B)$. NGF, NT-3, and NT-4 had no significant effects on RGC dendritic arbors. Plotted values represent the averages from five independent experiments; error bars indicate \pm SEM. Numbers within the bars indicate the total number $(n)$ of RGCs analyzed for each treatment. $* * * p<0.001$ as compared with control.

played distributions of dendritic complexities in which RGC DCI values ranged from very low to very high in each experimental treatment. The distribution of DCI values, however, revealed that altering BDNF levels regulated overall RGC dendritic complexity in vivo (Fig. 6C). Of RGCs exposed to exogenous BDNF, 65\% had DCI values in the least complex category (DCI $<250$ ) as compared with $27 \%$ of control RGCs and only $10 \%$ of RGCs exposed to anti-BDNF. Correspondingly, $27 \%$ of anti-BDNFtreated RGCs were in the most complex category (exhibited DCI values $>4250$ ), whereas only $12 \%$ of control and none of the BDNF-treated RGCs had DCI values in this highly complex category. Thus, this analysis of DCI distributions revealed that exogenous retinal BDNF skewed RGC complexities toward lower DCI values, whereas neutralizing endogenous retinal BDNF with antibodies skewed RGC dendritic complexity toward higher DCI values. Similar to our observations of primary dendrite number and secondary branching, neurotrophins other than BDNF did not exert significant effects on overall dendritic complexity. DCI distribution analysis revealed that the complexities of RGCs in NGF-, NT-3-, and NT-4-treated retinae varied slightly among 


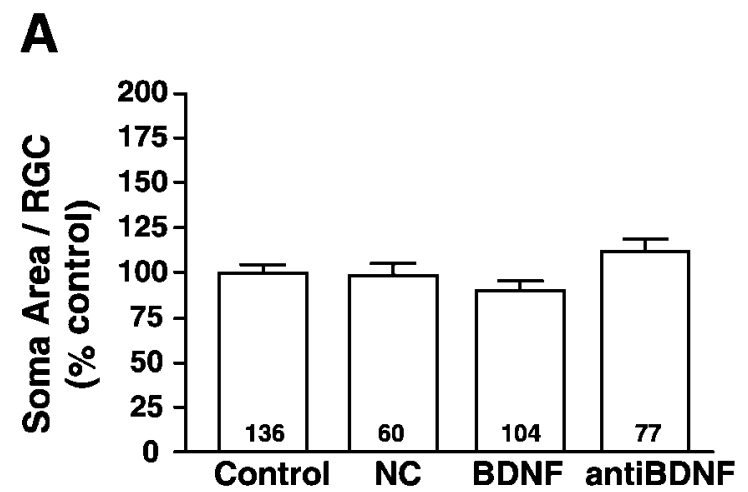

B

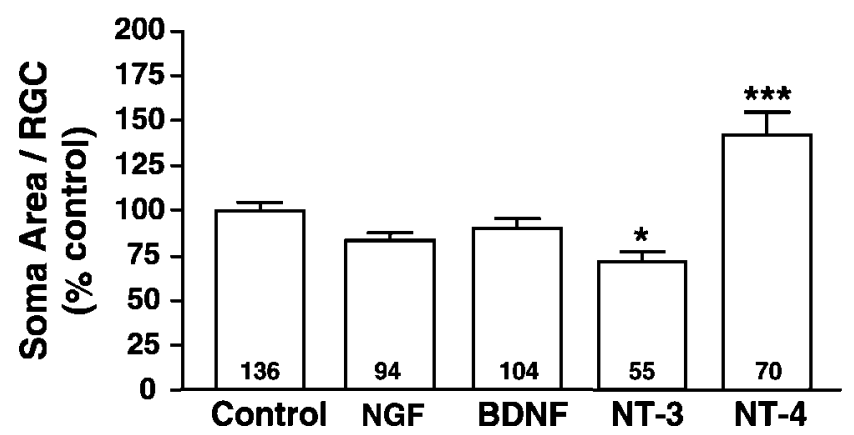

Figure 5. Neurotrophic regulation of RGC soma area. To determine whether retinal neurotrophins modulate aspects of RGC morphology in addition to dendritic arborization, we measured the soma area of RGCs in retinae treated with experimentally altered neurotrophin levels. The soma area of RGCs coincident with control-, BDNF-, or anti-BDNFtreated microspheres as well as RGCs not coincident (NC) with BDNFcoated microspheres were compared and found not to differ significantly from control $(A)$. Similarly, the effects of NGF, NT-3, and NT-4 on soma size also were compared $(B)$. BDNF and NGF did not alter soma size, but NT-3-treated RGCs had significantly smaller soma and NT-4-treated RGCs had significantly larger soma than control-treated RGCs, indicating that NT-3 and NT-4 exert opposing influences on soma size. Plotted values represent the averages from five independent experiments; error bars indicate \pm SEM. Numbers within the bars indicate the total number $(n)$ of RGCs analyzed for each treatment. ${ }^{* * *} p<0.001$ and $* p<0.05$ as compared with control.

each treatment, but DCI values generally resembled those of controls (distribution not shown; for morphologies and DCI values, see Fig. 2). Thus, only treatments that altered retinal BDNF levels evoked dramatic alterations in RGC dendritic complexity.

\section{Target-derived BDNF promotes RGC axonal arborization}

To compare the local effects of alterations in retinal BDNF levels on dendritic morphology with those of tectal BDNF on RGC axon morphologies, we experimentally altered neurotrophin levels in the Xenopus tectum during the developmental stages when RGC axons actively arborize (Fig. 7) (also see Cohen-Cory and Fraser, 1995). BDNF, control vehicle, or function-blocking BDNF antibodies were injected into the subpial space overlying the optic tectum to alter tectal neurotrophin levels (see Materials and Methods). The morphology of DiI-labeled RGC axon arbors was assessed immediately preceding and $24 \mathrm{hr}$ after tectal injections. The differences in total branch number between 0 and $24 \mathrm{hr}$ as well as the differences in axon arbor length at 0 and $24 \mathrm{hr}$ were used as a measure of change in axon arbor complexity (see Fig.
A

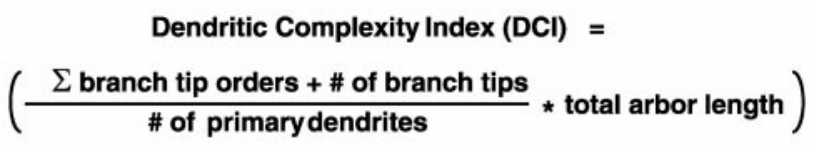

B

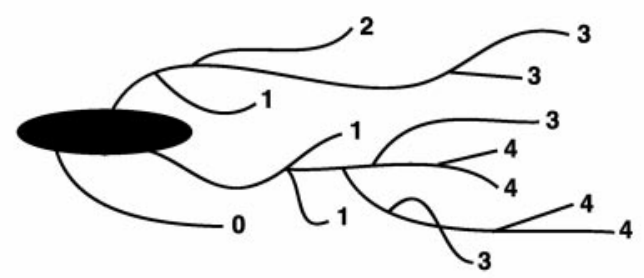

C

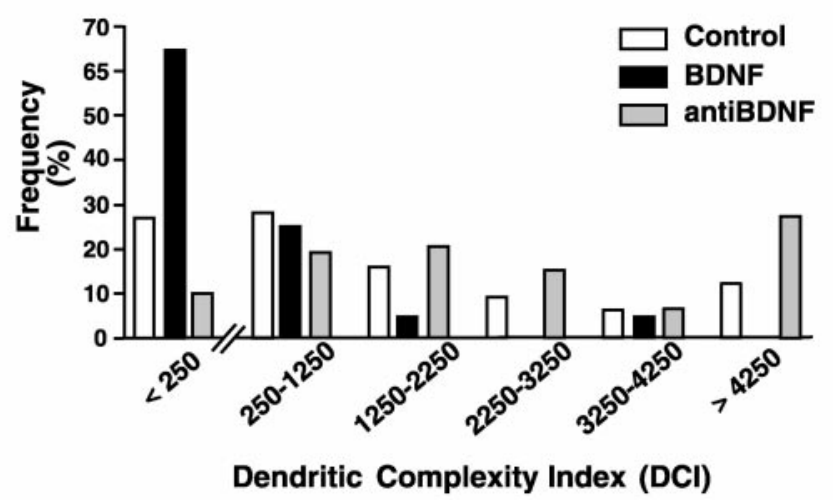

Figure 6. Retinal neurotrophin levels influence RGC dendritic complexity in vivo. To determine the influence of altering retinal neurotrophin levels on overall RGC dendritic morphology, we calculated a dendritic complexity index $(D C I)$ that numerically describes the overall dendritic arbor complexity for each RGC. $A$, The DCI calculation is based on the number of primary dendrites, total arbor length, and the number and order of branch tips (also see Materials and Methods for a description of the calculation). B, Diagram illustrating branch order analysis. Each branch tip is assigned a number (1-4) that equals the number of times a primary dendrite branches to produce the branch tip. $C$, DCI distribution analysis reveals different distributions of RGC complexities in control-, BDNF-, or anti-BDNF-treated retinae. RGC with simple, medium, and complex morphologies, however, are represented in all experimental conditions. Note that a majority of BDNF-treated RGCs fall into the simpler complexity group (DCI values of $<250$ ), whereas RGCs in anti-BDNF-treated retina have a skewed distribution toward larger DCI values than controls.

$6 A$ ). When compared with controls, axonal arbors became morphologically more elaborate over time in the BDNF-treated tadpoles and became simpler in those treated with anti-BDNF. After $24 \mathrm{hr}$ BDNF-treated arbors had significantly more branches $(\mathrm{BDNF}=6.8 \pm 0.87$ branches vs control $=2.5 \pm 0.4$ branches $)$ and increased their length significantly versus controls $(\mathrm{BDNF}=$ $177 \pm 25 \mu \mathrm{m}$ vs control $=107 \pm 14 \mu \mathrm{m})$. Neutralization of tectal BDNF via function-blocking BDNF antibodies resulted in axonal arbors that were less complex than controls at $24 \mathrm{hr}$ after treatment (increase in branch number, anti-BDNF $=0.6 \pm 0.5$ branches vs control $=2.5 \pm 0.4$ branches; increase in branch length, anti-BDNF $=69 \pm 19$ vs control $=107 \pm 17 \mu \mathrm{m})$. Taken together, these results indicate that endogenous tectal BDNF increases the complexity of axonal arbors, whereas retinal BDNF decreases dendritic complexity. 
A

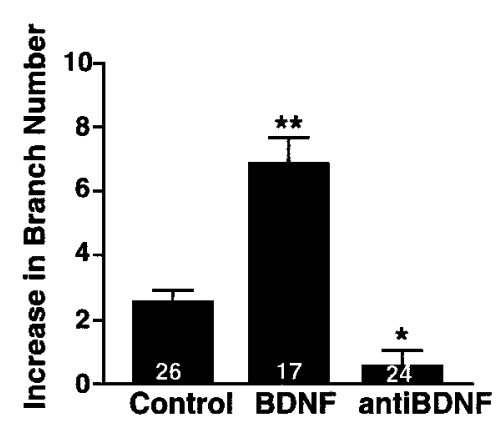

B

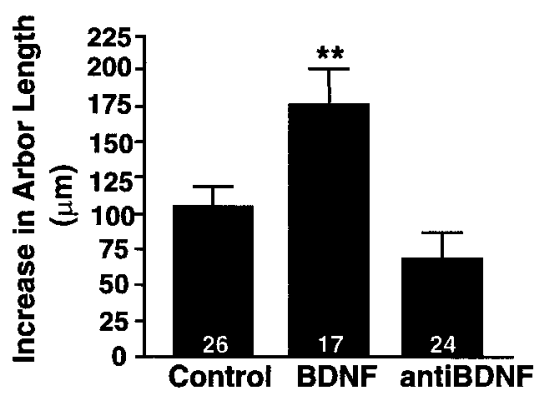

C
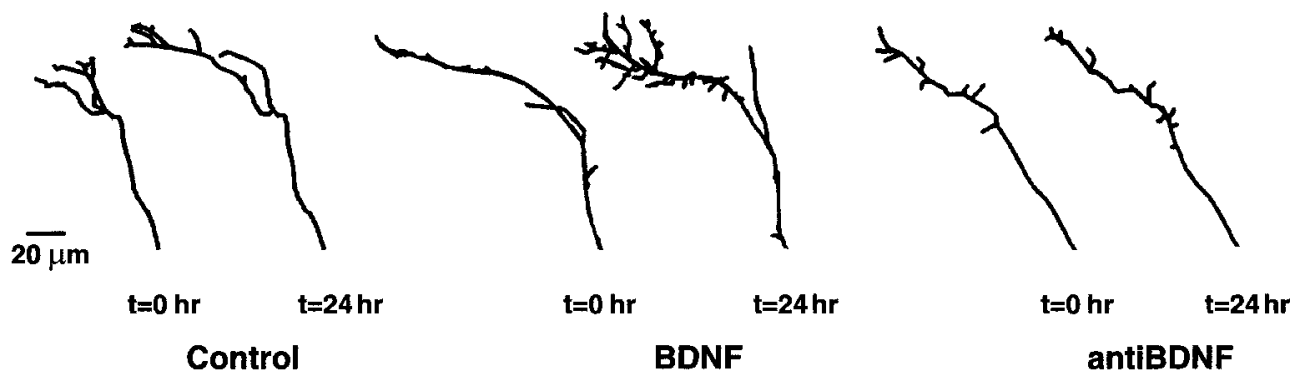

Figure 7. BDNF enhances RGC axonal arborization in vivo. Time-lapse analysis of DiI-labeled RGC axon arbors reveals that the morphology of RGC axonal arbors is influenced by tectal BDNF levels (also see Cohen-Cory and Fraser, 1995). $A$, $B$, The effects of BDNF and anti-BDNF on RGC axon arbor morphology after $24 \mathrm{hr}$ of treatment were evaluated by measuring quantitatively the total branch number $(A)$ and total arbor length $(B)$. Values are presented as the change from their initial value at the time of treatment. The significant increase in total branch number and arbor length elicited by BDNF reflects an overall increase in axon arbor complexity versus controls. Similarly, the significantly lower increase in branch number elicited by anti-BDNF indicates that neutralizing endogenous BDNF prevents the increase in axon terminal arbor complexity that normally occurs over time in normal tadpoles. Values represent the averages; error bars indicate \pm SEM. ${ }^{* *} p<0.01$ and ${ }^{*} p<0.05$ as compared with control. $C$, Tracings of representative axonal arbors before and $24 \mathrm{hr}$ after control, BDNF, or anti-BDNF injections illustrate the effects of each treatment on RGC axon arbor complexity. Controls include axons from tadpoles treated with vehicle solutions or with nonimmune IgGs. Scale bar, $20 \mu \mathrm{m}$.

\section{DISCUSSION}

BDNF expression in the developing retina and tectum suggests that BDNF may exert dual modes of action on RGC differentiation by acting both at the target and locally within the retina. BDNF is expressed at low levels in the retina at stage 39/40, coincident with the onset of RGC dendritic arborization, and peaks at stage 45 , when RGCs are actively elaborating axonal and dendritic arbors (Cohen-Cory and Fraser, 1994; Cohen-Cory et al., 1996). This report provides evidence that retinal BDNF significantly influences the development of RGC dendritic arbors in vivo. Surprisingly, application of exogenous BDNF to the retina during $\mathrm{RGC}$ dendritic arborization resulted in less complex RGC dendritic arbors. Exogenous BDNF significantly reduced RGC primary dendrite extension and secondary branching without influencing RGC soma size. Correspondingly, retinal applications of neutralizing BDNF antibodies resulted in more complex RGC dendritic arbors. In the developing Xenopus retina BDNF expression has been detected exclusively on RGCs, whereas both RGCs and amacrine cells express trkB (CohenCory and Fraser, 1994; Cohen-Cory et al., 1996). Thus, our results demonstrate that alterations in endogenous retinal BDNF levels result in alterations in RGC dendrite elaboration in vivo and support the idea that RGC-derived BDNF may act primarily in an autocrine manner to modulate RGC development and differentiation. The possibility still remains that BDNF also acts on other trkB-responsive retinal neurons, such as amacrine cells, and that amacrine cells in turn may act on RGCs, thereby indi- rectly altering RGC morphological differentiation. These two possibilities, however, are not mutually exclusive.

Although exposure to exogenous BDNF dramatically and significantly reduced the complexity of RGC dendritic arbors in vivo, some BDNF-treated RGCs elaborated complex dendritic arbors even in the presence of exogenous BDNF, suggesting that a subpopulation of RGCs is unaffected by retinal BDNF. In support of a nonresponsive RGC subpopulation, it has been shown that not all Xenopus RGCs express the trkB receptor and not all cultured RGCs respond to anti-BDNF (Cohen-Cory and Fraser, 1994; Cohen-Cory et al., 1996). Specific patterns of RGC dendritic arborization therefore might be achieved by precise local patterns of neurotrophin and receptor expression on individual RGCs. In this way, subtle neurotrophin sculpting of RGC dendritic morphology could produce the variety of RGC arbor morphologies that constitute the mature tadpole retinae (Sakaguchi et al., 1984). Our observations, however, do not rule out the possibility that all RGCs may respond to BDNF or anti-BDNF treatments, but with differing degrees of responsiveness. The variety of dendritic arbor complexities we observed in retinae exposed to either BDNF or anti-BDNF also may be attributable to different RGC maturities at the onset of treatment.

One indication that BDNF specifically influences RGC dendritic development arises from our observations that applications of anti-BDNF caused opposite effects on RGC dendritic arbor morphology than did applications of exogenous BDNF. When endogenous retinal BDNF was neutralized by function-blocking 
BDNF antibodies, RGCs elaborated more complex arbors than control RGCs. Experiments that significantly reduce an endogenous neurotrophin are necessary to confirm the role of that neurotrophin during development and to assess more directly whether exogenous neurotrophins mimic such effects (Shelton et al., 1995; McAllister et al., 1999). Two convenient methods to neutralize endogenous BDNF in vivo are available: trkB receptor bodies and specific BDNF function-blocking antibodies. Trk receptor bodies are fusion proteins of the ligand-binding domain of a specific trk receptor with the FC domain of human IgGs that bind available neurotrophin and thereby prevent neurotrophins from signaling via their trk receptors. Although this approach has provided supporting evidence for neurotrophic actions in vitro and in vivo, it also has caused controversy. Identical treatments with two different trkB receptor body preparations caused opposite effects on the same neuronal population (Frost et al., 1998). Another limitation of trkB receptor bodies is that ligand-specific neutralization cannot be achieved; trkB receptor bodies neutralize both NT-4 and BDNF. Function-blocking neurotrophin antibodies, however, specifically neutralize a single neurotrophin by binding endogenous neurotrophin and preventing it from interacting with all its receptors. The current study used a BDNF function-blocking antibody to neutralize BDNF in vivo without affecting retinal NT-4 levels. Therefore, our observations of the action of anti-BDNF support a role for endogenous retinal BDNF in modulating RGC dendritic morphology.

NT-4 and BDNF share the same specific receptor, trkB (Barbacid, 1994), yet in our study BDNF specifically inhibited dendritic complexity and NT-4 specifically increased soma size. This differential cellular response to two ligands that share a common receptor suggests that BDNF and NT-4 may be exerting their effects on RGC dendritic complexity and soma size via distinct receptors, receptor combinations, and/or intracellular signaling cascades. Other studies also have observed that trkB ligands can differentially influence specific aspects of neuronal morphology in the developing visual system. NT-4, but not BNDF, can prevent some of the effects of monocular deprivation on lateral geniculate neuron soma size (Riddle et al., 1995). Branching of Xenopus RGC axon arbors in vivo is promoted by BDNF but is unaffected by NT-4 (Cohen-Cory and Fraser, 1995). BDNF and NT-4 also have been shown to exert differential effects on cortical neuron dendritic morphology in vitro (McAllister et al., 1995, 1997).

In our study BDNF was the only neurotrophin to influence RGC dendritic morphology; NT-3 and NT-4 influenced RGC soma size without significantly influencing dendritic complexity. RGC soma treated with NT-3 were significantly smaller than controls, whereas NT-4-treated RGCs had significantly larger soma. NT-4 has been shown to prevent thalamic neuron soma shrinkage resulting from unbalanced input activity (Riddle et al., 1995). NT-3 is known to promote early RGC differentiation, converting retinal progenitors into differentiated RGCs (de la Rosa et al., 1994; Bovolenta et al., 1996). Thus, our results agree with the observations that NT-4 may affect soma size and that NT-3 may act as a differentiation signal for RGCs. Therefore, it is likely that individual neurotrophins differentially modulate distinct components of RGC differentiation, such as soma size and dendritic elaboration. In the mature retina, RGC subtype classifications are based on both dendritic morphology and RGC soma size (Wingate and Thompson, 1994). Neurotrophins therefore may act as determinants of RGC subtype specification by modulating distinct aspects of differentiation. The expression patterns of neurotrophins and their receptors within the devel- oping retina (Cohen-Cory and Fraser, 1994; Hutson and Bothwell, 1998; Karlsson et al., 1998) (for review, see von Bartheld, 1998) further support local roles for these neurotrophins and make it plausible that alterations in retinal neurotrophin levels may affect specific aspects of retinal development in vivo.

The neurotrophins can exert a wide variety of influences on neuronal morphology. Most reported neurotrophic effects on dendritic morphology analyzed in culture indicate that individual neurotrophins promote the arborization of specific neuronal populations (Cohen-Cory et al., 1991; McAllister et al., 1996). For example, in slice cultures of ferret visual cortex the pyramidal neurons from different cortical layers respond differentially to neurotrophins (McAllister et al., 1995, 1996, 1997). Individual neurotrophins exert distinct effects on either basal or apical dendrites, with each neurotrophin eliciting a unique pattern of dendritic morphologies for neurons within a single cortical layer. Although in most instances neurotrophic factors promote dendritic arborization, in some neuronal populations they inhibit neuritogenesis. The cytokine leukemia inhibitory factor (LIF) and ciliary neurotrophic factor (CNTF) have been shown to reduce neurite outgrowth from dissociated sympathetic neurons (Guo et al., 1999), and BDNF has been shown to reduce basal, but not apical, dendritic complexity of layer 6 cortical pyramidal neurons in culture (McAllister et al., 1995, 1997). Thus, combining our observations that BDNF reduces RGC dendritic complexity in vivo with previous in vitro studies reveals a novel, additional role for neurotrophic factors as negative regulators of neuronal arborization.

Previous studies demonstrated that Xenopus RGCs initiate primary dendrites and elaborate dendritic arbors by targetindependent mechanisms (Holt, 1989; Sakaguchi, 1989). Short, simple RGC dendrites can be observed before the first RGC axons reach the optic tectum, indicating that dendritic initiation is independent of target-derived cues (Holt, 1989). Further, the morphology and subtype specification of RGC dendritic arbors in ectopically transplanted eyes are indistinguishable from controls, indicating that even in the complete absence of target interactions RGC dendritic arborization occurs normally (Sakaguchi, 1989). Most BDNF in the retinal ganglion cell layer is not of tectal origin, although RGCs are capable of retrogradely transporting tectal BDNF, suggesting that most retinal BDNF is produced locally within the retina (Herzog and von Bartheld, 1998). Thus, it is likely that retinal-derived BDNF exerts much stronger local influence within the retina than does tectal BDNF. This report demonstrates that alterations in retinal BDNF levels before target innervation cause alterations in RGC dendritic morphology, suggesting that BDNF can act locally within the retina to influence dendritic development well before RGC axons reach the tectum where they have the opportunity to interact with targetderived BDNF. That local and target-derived BDNF exert distinct roles during RGC development is supported by our unique observation that this neurotrophin is capable of differentially modulating axonal versus dendritic arborization within a single neuronal population. Alterations in tectal BDNF levels significantly altered axon arbor morphology, whereas alterations in BDNF retinal levels altered dendritic morphology, suggesting local roles for BDNF in both locations. The possibility still exists that target-derived BDNF also could contribute to the local effects of BDNF on dendritic morphology, although RGC dendrites begin to develop before their axons reach the tectum. The experiments in this study did not eliminate this possibility. To what extent target-derived BDNF contributes to RGC dendritic 
differentiation and, conversely, retinal-derived BDNF contributes to RGC axon arborization remains to be determined.

In most vertebrate species, developing RGCs initially elaborate exuberant dendritic arbors by extending an excess number of branches that later are remodeled to achieve their final morphological and maturational state (Vanselow et al., 1990; Wong et al., 1991; Yamasaki and Ramoa, 1993). Combinations of intrinsic retinal influences and target interactions are thought to play roles in determining RGC dendritic form (for review, see Wingate, 1996). Interactions that depend on neuronal activity only slightly influence the intrinsic RGC dendritic remodeling program. For example, blocking action potential activity does not prevent the pruning and remodeling of exuberant RGC dendritic arbors (Wong et al., 1991; Campbell et al., 1997) but increases RGC axonal arbor complexity (Sretavan et al., 1988). Only manipulations in RGC density that are independent of target interactions significantly influence RGC dendritic elaboration and remodeling (Troilo et al., 1996). These observations suggest that intrinsic signals within the developing retina exert significant control on RGC dendritic elaboration and remodeling. Our results demonstrating that alterations in retinal BDNF levels before full differentiation of RGC dendritic arbors (and before the peak in endogenous BDNF expression) lead to alterations in RGC dendritic morphology suggest that endogenous retinal BDNF may be one of the fine regulatory signals that locally control RGC dendritogenesis and remodeling in vivo. Moreover, the observation that RGCs express both BDNF and trkB during this developmental period suggests that a single neuronal population can finely tune its morphological differentiation program via the coordinated action of neurotrophic factors.

Our in vivo observations that BDNF differentially modulates RGC axonal and dendritic arbor morphology in the developing visual system raise the intriguing possibility that neurotrophic signaling at the axon terminal of a neuron can differ dramatically from signaling at its dendrites. The mechanism underlying this differential response to BDNF within a single neuronal population is unknown. Within the developing visual system it is likely that BDNF acts in coordination with other cues to direct both axonal and dendritic arborization differentially. RGC axons and dendrites synapse with distinctly different neuronal populations and thus may be exposed to different cues that locally can mediate axonal and dendritic responses to BDNF in a differential manner. Not only are the environments that the axon and dendrites of an RGC encounter different, but the complement of cell surface receptors may differ between its axons and dendrites. Recent observations that neurotransmitter receptors can be differentially targeted to axons and dendrites (Stowell and Craig, 1999) suggest that selective delivery of cell surface receptors could explain the ability of a neuron to respond differentially at its axons and dendrites. Similarly, differential expression of intracellular signal transduction machinery in axons versus dendrites also might mediate their differential responses to a single cue. Recent work in vitro has demonstrated that cyclic nucleotides are involved in intracellular signaling by chemoattractive and chemorepulsive cues. Pharmacological alterations in cyclic nucleotides caused growth cones to switch their response to a chemoattractive molecule from attraction into repulsion (Song et al., 1997, 1998). Thus, differentially regulated alterations in intracellular signaling mechanisms have the potential to cause opposing cellular responses within the axons and dendrites of a single neuron. Precise patterns of neurotrophin expression, receptor localization, and intracellular signaling mechanisms thereby may exert spatial and temporal control over neuronal morphology and profoundly influence nervous system development.

\section{REFERENCES}

Barbacid M (1994) The Trk family of neurotrophin receptors. J Neurobiol 25:1386-1403.

Barbacid M (1995) Neurotrophic factors and their receptors. Curr Opin Cell Biol 7:148-155.

Barde YA (1994) Neurotrophins: a family of proteins supporting the survival of neurons. Prog Clin Biol Res 390:45-56.

Berninger B, Poo M (1996) Fast actions of neurotrophic factors. Curr Opin Neurobiol 6:324-330.

Bothwell M (1995) Functional interactions of neurotrophins and neurotrophin receptors. Annu Rev Neurosci 18:223-253.

Bovolenta P, Frade JM, Marti E, Rodriguez-Pena MA, Barde YA, Rodriguez-Tebar A (1996) Neurotrophin-3 antibodies disrupt the normal development of the chick retina. J Neurosci 16:4402-4410.

Cabelli RJ, Hohn A, Shatz CJ (1995) Inhibition of ocular dominance column formation by infusion of NT-4/5 or BDNF. Science 267:1662-1666.

Campbell G, Ramoa AS, Stryker MP, Shatz CJ (1997) Dendritic development of retinal ganglion cells after prenatal intracranial infusion of tetrodotoxin. Vis Neurosci 14:779-788.

Cohen-Cory S, Fraser SE (1994) BDNF in the development of the visual system of Xenopus. Neuron 12:747-761.

Cohen-Cory S, Fraser SE (1995) Effects of brain-derived neurotrophic factor on optic axon branching and remodeling in vivo. Nature 378:192-196.

Cohen-Cory S, Dreyfus CF, Black IB (1991) NGF and excitatory neurotransmitters regulate survival and morphogenesis of cultured cerebellar Purkinje cells. J Neurosci 11:462-471.

Cohen-Cory S, Escandon E, Fraser SE (1996) The cellular patterns of BDNF and trkB expression suggest multiple roles for BDNF during Xenopus visual system development. Dev Biol 179:102-115.

de la Rosa EJ, Arribas A, Frade JM, Rodriguez-Tebar A (1994) Role of neurotrophins in the control of neural development: neurotrophin-3 promotes both neuron differentiation and survival of cultured chick retinal cells. Neuroscience 58:347-352.

Escandon E, Soppet D, Rosenthal A, Mendoza-Ramirez JL, Szonyi E, Burton LE, Henderson CE, Parada LF, Nikolics K (1994) Regulation of neurotrophin receptor expression during embryonic and postnatal development. J Neurosci 14:2054-2068.

Frost DO, Ma Y-T, Pollock G, Robichon R, Lyons WE, Ambalavanar R, Kaplan DR (1998) Role of trkB ligands in the developmental death of hamster retinal ganglion cells. Soc Neurosci Abstr 24:294.

Garner AS, Menegay HJ, Boeshore KL, Xie XY, Voci JM, Johnson JE, Large TH (1996) Expression of TrkB receptor isoforms in the developing avian visual system. J Neurosci 16:1740-1752.

Ghosh A, Carnahan J, Greenberg ME (1994) Requirement for BDNF in activity-dependent survival of cortical neurons. Science 263:1618-1623.

Goodman CS, Shatz CJ (1993) Developmental mechanisms that generate precise patterns of neuronal connectivity. Cell [Suppl] 72:77-98.

Guo X, Chandrasekaran V, Lein P, Kaplan PL, Higgins D (1999) Leukemia inhibitory factor and ciliary neurotrophic factor cause dendritic retraction in cultured rat sympathetic neurons. J Neurosci 19:2113-2121.

Hallbook F, Backstrom A, Kullander K, Ebendal T, Carri NG (1996) Expression of neurotrophins and Trk receptors in the avian retina. J Comp Neurol 364:664-676.

Herzog KH, von Bartheld CS (1998) Contributions of the optic tectum and the retina as sources of brain-derived neurotrophic factor for retinal ganglion cells in the chick embryo. J Neurosci 18:2891-2906.

Holt CE (1989) A single-cell analysis of early retinal ganglion cell differentiation in Xenopus: from soma to axon tip. J Neurosci 9:3123-3145.

Hutson LD, Bothwell MA (1998) Neurotrophin signaling through p75NTR promotes cell survival during development of the Xenopus retina. Soc Neurosci Abstr 24:43.

Jelsma TN, Friedman HH, Berkelaar M, Bray GM, Aguayo AJ (1993) Different forms of the neurotrophin receptor TrkB mRNA predominate in rat retina and optic nerve. J Neurobiol 24:1207-1214.

Karlsson M, Clary DO, Lefcort FB, Reichardt LF, Karten HJ, Hallbook F (1998) Nerve growth factor receptor TrkA is expressed by horizontal and amacrine cells during chicken retinal development. J Comp Neurol 400:408-416. 
Keller R (1991) Early embryonic development of Xenopus laevis. In: Xenopus laevis: practical uses in cell and molecular biology (Kay BK, Peng HB, eds), pp 102-116. San Diego: Academic.

McAllister AK, Lo DC, Katz LC (1995) Neurotrophins regulate dendritic growth in developing visual cortex. Neuron 15:791-803.

McAllister AK, Katz LC, Lo DC (1996) Neurotrophin regulation of cortical dendritic growth requires activity. Neuron 17:1057-1064.

McAllister AK, Katz LC, Lo DC (1997) Opposing roles for endogenous BDNF and NT-3 in regulating cortical dendritic growth. Neuron 18:767-778.

McAllister AK, Katz LC, Lo DC (1999) Neurotrophins and synaptic plasticity. Annu Rev Neurosci 22:295-318.

Nawa H, Carnahan J, Gall C (1995) BDNF protein measured by a novel enzyme immunoassay in normal brain and after seizure: partial disagreement with mRNA levels. Eur J Neurosci 7:1527-1535.

Nieuwkoop PD, Faber J (1956) Normal table of Xenopus development. Amsterdam: Elsevier.

O'Rourke N, Cline HT, Fraser SE (1994) Rapid remodeling of retinal arbors in the tectum with and without blockade of synaptic transmission. Neuron 12:921-934.

Perez MT, Caminos E (1995) Expression of brain-derived neurotrophic factor and of its functional receptor in neonatal and adult rat retina. Neurosci Lett 183:96-99.

Riddle DR, Lo DC, Katz LC (1995) NT-4-mediated rescue of lateral geniculate neurons from effects of monocular deprivation. Nature 378:189-191.

Riddle DR, Katz LC, Lo DC (1997) Focal delivery of neurotrophins into the central nervous system using fluorescent latex microspheres. Biotechniques 23:928-937.

Rodriguez-Tebar A, Jeffrey PL, Thoenen H, Barde YA (1989) The survival of chick retinal ganglion cells in response to brain-derived neurotrophic factor depends on their embryonic age. Dev Biol 136:296-303.

Sakaguchi DS (1989) The development of retinal ganglion cells deprived of their targets. Dev Biol 134:103-111.

Sakaguchi DS, Murphey RK, Hunt RK, Tompkins R (1984) The development of retinal ganglion cells in a tetraploid strain of Xenopus laevis: a morphological study utilizing intracellular dye injection. J Comp Neurol 224:231-251.
Shelton DL, Sutherland J, Gripp J, Camerato T, Armanini MP, Phillips HS, Carroll K, Spencer S, Levinson AD (1995) Human trks: molecular cloning, tissue distribution, and expression of extracellular domain immunoadhesins. J Neurosci 15:477-491.

Snider WD, Lichtman JW (1996) Are neurotrophins synaptotrophins? Mol Cell Neurosci 7:433-442.

Song HJ, Ming GL, Poo MM (1997) cAMP-induced switching in turning direction of nerve growth cones. Nature 388:275-279.

Song H, Ming G, He Z, Lehmann M, Tessier-Lavigne M, Poo M (1998) Conversion of neuronal growth cone responses from repulsion to attraction by cyclic nucleotides. Science 281:1515-1518.

Sretavan DW, Shatz CJ, Stryker MP (1988) Modification of retinal ganglion cell axon morphology by prenatal infusion of tetrodotoxin. Nature 336:468-471.

Stowell JN, Craig AM (1999) Axon/dendrite targeting of metabotropic glutamate receptors by their cytoplasmic carboxy-terminal domains. Neuron 22:525-536.

Troilo D, Xion M, Crowley JC, Finlay BL (1996) Factors controlling the dendritic arborization of retinal ganglion cells. Vis Neurosci 13:721-733.

Vanselow J, Dutting D, Thanos S (1990) Target dependence of chick retinal ganglion cells during embryogenesis: cell survival and dendritic development. J Comp Neurol 295:235-247.

von Bartheld CS (1998) Neurotrophins in the developing and regenerating visual system. Histol Histopathol 13:437-459.

Wingate RJ (1996) Retinal ganglion cell dendritic development and its control: filling the gaps. Mol Neurobiol 12:133-144.

Wingate RJ, Thompson ID (1994) Targeting and activity-related dendritic modification in mammalian retinal ganglion cells. J Neurosci 14:6621-6637.

Witte S, Stier H, Cline HT (1996) In vivo observations of time course and distribution of morphological dynamics in Xenopus retinotectal axon arbors. J Neurobiol 31:219-234.

Wong RO, Herrmann K, Shatz CJ (1991) Remodeling of retinal ganglion cell dendrites in the absence of action potential activity. J Neurobiol 22:685-697.

Yamasaki EN, Ramoa AS (1993) Dendritic remodeling of retinal ganglion cells during development of the rat. J Comp Neurol 329:277-289. 